\title{
TLR7 activation in epilepsy of tuberous sclerosis complex
}

\author{
Alan A. Dombkowski ${ }^{1,6}$ - Daniela Cukovic ${ }^{1}$. Shruti Bagla ${ }^{1} \cdot$ McKenzie Jones $^{1} \cdot$ Joseph A. Caruso $^{2}$. \\ Harry T. Chugani ${ }^{3,4}$. Diane C. Chugani, ${ }^{3,5}$
}

Received: 31 May 2018 / Revised: 30 August 2019 / Accepted: 5 September 2019 / Published online: 11 September 2019 (c) The Author(s) 2019

\begin{abstract}
Background Neuroinflammation and toll-like receptors (TLR) of the innate immune system have been implicated in epilepsy. We previously reported high levels of microRNAs miR-142-3p and miR-223-3p in epileptogenic brain tissue resected for the treatment of intractable epilepsy in children with tuberous sclerosis complex (TSC). As miR-142-3p has recently been reported to be a ligand and activator of TLR7, a detector of exogenous and endogenous single-stranded RNA, we evaluated TLR7 expression and downstream IL23A activation in surgically resected TSC brain tissue.

Methods Gene expression analysis was performed on cortical tissue obtained from surgery of TSC children with pharmacoresistent epilepsy. Expression of TLRs 2, 4 and 7 was measured using NanoString nCounter assays. Real-time quantitative PCR was used to confirm TLR7 expression and compare TLR7 activation, indicated by IL-23A levels, to levels of miR-142-3p. Protein markers characteristic for TLR7 activation were assessed using data from our existing quantitative proteomics dataset of TSC tissue. Capillary electrophoresis Western blots were used to confirm TLR7 protein expression in a subset of samples. Results TLR7 transcript expression was present in all TSC specimens. The signaling competent form of TLR7 protein was detected in the membrane fraction of each sample tested. Downstream activation of TLR7 was found in epileptogenic lesions having elevated neuroinflammation indicated by clinical neuroimaging. TLR7 activity was significantly associated with tissue levels of miR-142-3p.

Conclusion TLR7 activation by microRNAs may contribute to the neuroinflammatory cascade in epilepsy in TSC. Further characterization of this mechanism may enable the combined of use of neuroimaging and TLR7 inhibitors in a personalized approach towards the treatment of intractable epilepsy.
\end{abstract}

Keywords Neuroinflammation $\cdot$ Epilepsy $\cdot$ Tuberous sclerosis complex $\cdot$ Toll-like receptor $\cdot$ MicroRNA $\cdot$ AMT-PET

Responsible Editor: Thiago Mattar Cunha.

Alan A. Dombkowski

domski@wayne.edu

1 Department of Pediatrics, Wayne State University School of Medicine, Detroit, MI, USA

2 Institute of Environmental Health Sciences, Wayne State University, Detroit, MI 48201, USA

3 Katzin Diagnostic and Research PET/MR Center, Nemours/Alfred I. duPont Hospital for Children, Wilmington, DE, USA

4 Department of Neurology, Thomas Jefferson University, Philadelphia, PA, USA

5 Departments of Communication Sciences and Disorders, and Chemistry and Biochemistry, University of Delaware, Newark, DE, USA

6 Children's Hospital of Michigan, Clinical Pharmacology Room 3L22, 3901 Beaubien Blvd., Detroit, MI 48201, USA

\section{Introduction}

A growing body of evidence suggests that a 'vicious cycle' exists between seizures and neuroinflammation in some forms of epilepsy. In this scenario, a central nervous system insult due to injury, genetic alteration, or infection causes an initial onset of seizures that triggers a neuroinflammatory cascade, which further contributes to recurrent seizures [1]. A clinical biomarker associated with neuroinflammation and having nearly $100 \%$ specificity for identifying epileptogenic lesions is positron emission tomography (PET) imaging using the tracer $\alpha$-methyl-L-tryptophan (AMT) [2]. Cellular uptake of AMT is increased in tissue where the kynurenine pathway (KP) of tryptophan metabolism is activated by neuroinflammatory signaling, primarily through induction of indoleamine 2,3-dioxygenase (IDO1) and/or tryptophan 
Table 1 Patient demographics and specimen details

\begin{tabular}{|c|c|c|c|c|c|c|c|c|}
\hline Sample & Age & Gender & Hemisphere & Mutation & Location & Group & Lesion & $\overline{\text { Assays }}$ \\
\hline L1412RK-C & 2 years & M & Right & TSC2 & Frontal & Normal & Non-tuber & $a-d$ \\
\hline I1710AC-B & 5 years & M & Left & TSC2 & Frontal parietal & Normal & Non-tuber & $a-d$ \\
\hline I1914MC-C & 13 months & $\mathrm{F}$ & Left & TSC2 & Parietal & Normal & Non-tuber & $a-d$ \\
\hline E0214IM-A & 3 years & M & Right & TSC2 & Parietal & Normal & Non-tuber & $\mathrm{a}-\mathrm{d}$ \\
\hline E0214IM-B & 3 years & M & Right & TSC2 & Frontal & Normal & Non-tuber & $\mathrm{e}$ \\
\hline K2213NN-A & 6 years & M & Right & TSC2 & Occipital & Normal & Non-tuber & $\mathrm{e}$ \\
\hline 92804-B & 7.5 years & $\mathrm{F}$ & Left & Unknown & Frontal & $\mathrm{NC}$ & Tuber & $a-d^{*}$ \\
\hline 83002-A & 3 years & $\mathrm{F}$ & Left & TSC2 & Temporal & $\mathrm{NC}$ & Tuber & $\mathrm{a}-\mathrm{e}^{*}$ \\
\hline L1412RK-B & 2 years & M & Right & TSC2 & Frontal & $\mathrm{NC}$ & Tuber & $\mathrm{a}-\mathrm{d}^{*}$ \\
\hline I1710AC-C & 5 years & M & Left & $\mathrm{TSC} 2$ & Frontal & $\mathrm{NC}$ & Tuber & $a-d^{*}$ \\
\hline 81603-A & 8 years & M & Left & TSC2 & Temporal & $\mathrm{NC}$ & Tuber & $a-d^{*}$ \\
\hline G2710CC-A & 2 years & M & Right & TSC2 & Temporal & $\mathrm{NC}$ & Tuber & $\mathrm{e}$ \\
\hline G2710CC-B & 2 years & M & Right & TSC2 & Occipital & $\mathrm{OC}$ & Tuber & $\mathrm{a}-\mathrm{e}^{*}$ \\
\hline I1710AC-A & 5 years & M & Left & TSC2 & Frontal & $\mathrm{OC}$ & Tuber & $a-d^{*}$ \\
\hline 92804-A & 7.5 years & $\mathrm{F}$ & Left & Unknown & Frontal & $\mathrm{OC}$ & Tuber & $\mathrm{a}-\mathrm{d}^{*}$ \\
\hline J1513AB-B & 11 years & $\mathrm{F}$ & Left & TSC2 & Frontal parietal & $\mathrm{OC}$ & Tuber & $a-d^{*}$ \\
\hline 83002-D & 3 years & $\mathrm{F}$ & Left & TSC2 & Temporal & $\mathrm{OC}$ & Tuber & $\mathrm{e}$ \\
\hline F0508-B & 9 months & M & Left & TSC2 & Occipital & $\mathrm{OH}$ & Tuber & d, e \\
\hline L1412RK-A & 2 years & M & Right & TSC2 & Frontal & $\mathrm{OH}$ & Tuber & $a-d^{*}$ \\
\hline 81603-B & 8 years & M & Left & TSC2 & Frontal & $\mathrm{OH}$ & Tuber & $a-d^{*}$ \\
\hline F0508-A & 9 months & M & Left & TSC2 & Central frontal & $\mathrm{OH}$ & Tuber & $a-d^{*}$ \\
\hline 83002-B & 3 years & $\mathrm{F}$ & Left & TSC2 & Parietal & $\mathrm{OH}$ & Tuber & $a-e^{*}$ \\
\hline
\end{tabular}

a: miR-142-3p qPCR, b: IL23A qPCR, c: TLR7 qPCR, d: TLR NanoString, e: Western

*miR-142-3p qPCR data from [6]

2,3-dioxygenase (TDO2), rate-limiting enzymes of the pathway [3-5].

Tuberous sclerosis complex (TSC) arises due to genetic mutations that cause lesions in a number of organs, including the brain (tubers). Some cortical tubers trigger the onset of seizures (epileptogenic), while others do not. We recently reported elevation of several microRNAs in epileptogenic tubers from TSC patients [6]. Among these microRNAs, expression of miR-142-3p and miR-223-3p was strikingly correlated with AMT uptake, suggesting neuroinflammatory relevance. Studies have shown intercellular signaling mediated by cellular secretion of miR-142-3p in exosomes. A recent report found that miR-142-3p is a ligand and potent activator of the TLR7 receptor [7]. Additionally, others have shown that TLR7 can induce IDO1 and the KP [8]. Together, these findings suggest that the TLR7 receptor may be activated by the high level of miR-142-3p found in AMT-hot tissue, and miR-142-3p may play a role in neuroinflammation and KP induction in epileptogenic lesions. Currently, the role of TLR7 in epilepsy is unknown. Here, we sought to determine if TLR7 is expressed in TSC brain tissue and if miR-142-3p levels are associated with the receptor's activation as indicated by IL-23A expression, a marker of TLR7 activation [9]. We also measured expression of TLRs 2 and
4, which have been reported increased in TSC and can act synergistically with TLR7 $[10,11]$. This study has considerable translational relevance because TLR7 can be inhibited with existing drugs that are used to treat several autoimmune disorders.

\section{Methods}

\section{Tissue specimens}

Table 1 provides demographic data for each tissue specimen analyzed in this study and the assays performed on each specimen. Seizure onset and AMT status were determined as described in [6]. The amount of tissue available for each specimen is typically very limited and restricts the number of assays available for some samples. Informed consent was obtained from all participants, and the study was completed under Institutional Review Board \#043515MP4E.

\section{Real-time quantitative PCR (qPCR) of TSC specimens}

Gene expression analysis was performed using surgically resected TSC tissue, as indicated in Table 1. Of these, 
miR-142-3p data from a previous study were used for 13 specimens [6], representing three types of TSC tubers: seizure onset/AMT-hot (OH), onset/AMT-cold (OC), and nononset/AMT-cold (NC), where "hot" indicates elevated AMT uptake detected in clinical AMT-PET imaging and "onset" indicates epileptogenic activity detected by clinical electrocorticography. These specimens were supplemented by four additional control samples of non-tuber cortical tissue from TSC patients and several additional tuber specimens to enable Western blotting.

Gene expression qPCR for TLR7 and IL-23A was performed as previously described [6], using 2XPowerUp SYBRGreen master mix and the following primers:

\section{TLR7/forward: 5'GCTGATCTTGGCACCTCTC3'}

TLR7/reverse: 5'TGTCCACATTGGAAACACCATT3' IL-23A/forward: 5'CTCAGTGCCAGCAGCTTTC 3' IL-23A/reverse: 5'CCACACTGGATATGGGGAAC 3'

MicroRNA qPCR was performed for miR-142-3p on the four control samples, as described in [6], and miR-142-3p data for the other 13 specimens were utilized from [6].

\section{NanoString expression assay of TLRs}

Measurement of TLR 2, 4, and 7 levels was performed using the nCounter human neuroinflammation panel v1.0. For each sample as indicated in Table 1, $100 \mathrm{ng}$ of total RNA was used as input to the hybridization, performed at $65{ }^{\circ} \mathrm{C}$ for $17 \mathrm{~h}$. Data were background subtracted by the geometric mean of negative controls and normalized by the geometric mean of positive controls using nSolver v4.0.

\section{Capillary electrophoresis immunoblotting of TLR7}

Membrane and cytosolic protein fractions were isolated from frozen tissue using the Qiagen Qproteome Cell Compartment kit [12] and approximately 20-30 mg of frozen brain tissue. The brain tissue was disrupted in $500-\mu l$ lysis buffer supplemented with Protease Inhibitor Solution using a TissueRuptor (Qiagen) for $5 \mathrm{~s}$ at the lowest speed followed by QIAshredder homogenizer step. Subcellular fractionation of the brain tissue samples was conducted according to the vendor's protocol.

Capillary electrophoresis immunoblotting was performed by RayBiotech (Norcross, GA). Antibodies for TLR7 (Santa Cruz SC-57463) and calnexin (RayBiotech) were used. Each sample was loaded at $0.2 \mathrm{mg} / \mathrm{mL}$.

\section{Protein markers of TLR7 activation}

A list of proteins characteristically induced by TLR7 activation was obtained from Figure 9c of [13]. Quantitative MS/
MS spectra were available for nine of these proteins in our previous dataset comparing four onset/AMT-hot tubers to four non-tuber controls [14]. For each protein, the difference in expression was calculated between onset/hot tubers and controls.

\section{Statistical analysis}

Analysis was performed with JMP14. Levene's test for equality of variance was used to compare the variance between categories. No significance in the difference of variance was detected; therefore, we assumed homogeneity of variance. The relationship of IL-23A expression to AMT uptake and seizure status was investigated using two-way ANOVA with factors specified as: AMT uptake (hot/cold) and seizure status (non-onset/onset). Since there were only two levels for each factor, a post hoc test was not needed [15]. Regression analysis of IL-23A and miR-142-3p expression was performed using least squares. The significance of change in expression for TLR7 and NF-kB target proteins was performed using one-sample $t$ test of $\log$ (tuber/control) against zero (two-tailed). Results were considered significant at $P \leq 0.05$ for all statistical tests.

\section{Results}

Gene transcripts of TLRs 2, 4, and 7 were present in each TSC tissue specimen (Fig. 1a), but levels were not significantly associated with specimen categories: onset/AMT-hot tubers $(\mathrm{OH})$; onset/AMT-cold (OC); non-onset/AMT-cold (NC); and non-tuber control (Normal/NT). Gene expression of TLR7 was analyzed using two independent technologies and results were concordant $(P=0.0162)$. TLR7 protein expression was detected using capillary electrophoresis immunoblotting of membrane and cytosolic fractions from two specimens of each category (Fig. 1b). Precursor TLR7 undergoes enzymatic proteolysis to produce a signalingcompetent C-terminal fragment ( $\sim 60 \mathrm{kDa})$ that accumulates within endosomes, and is necessary and sufficient for TLR7 receptor signaling [16-18]. We found the signaling-competent form of TLR7 in the membrane fraction of each cortex specimen, as expected for endosomal accumulation.

IL-23A expression is a marker of TLR7 activation. ANOVA analysis revealed that IL-23A is significantly associated with AMT uptake (Fig. 2a), with higher expression found in AMT-hot specimens. This observation links TLR7 activation to neuroinflammation indicated by AMT uptake. Since miR-142-3p is a known ligand and activator of TLR7 [7], we investigated if IL-23A expression in these tissues is correlated to tissue-matched miR-142-3p levels. We applied regression analysis and found that miR-142-3p is a significant predictor of IL-23A expression, and a positive 

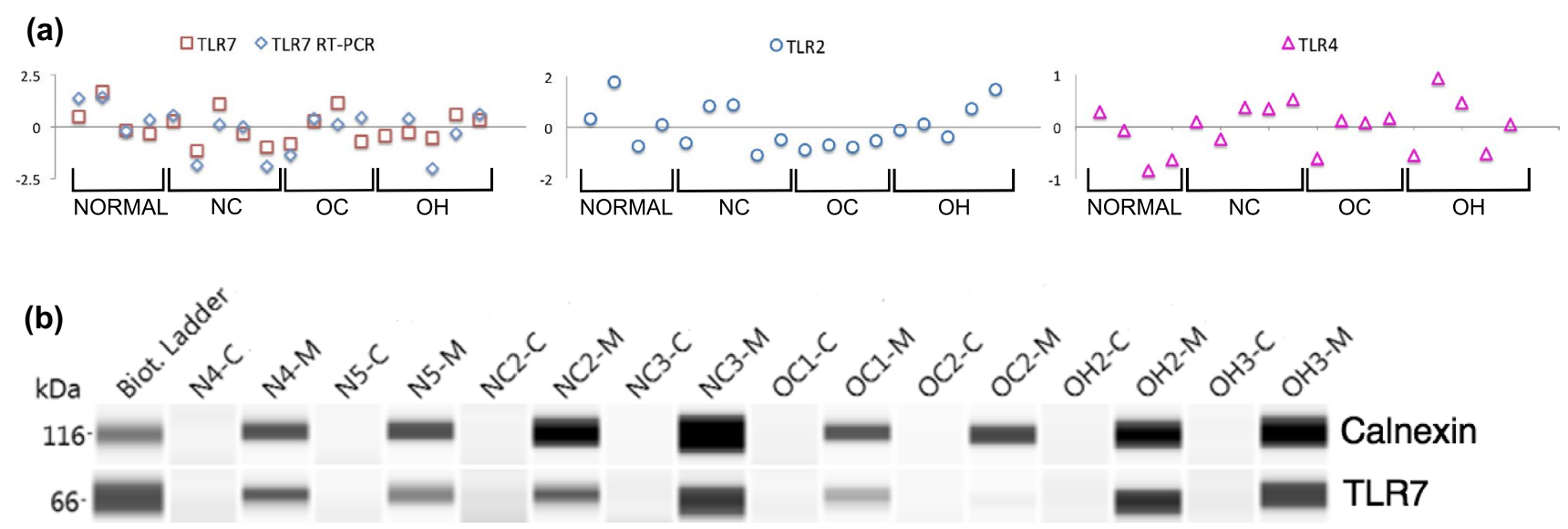

Fig. 1 TLR7 transcript and protein are expressed in TSC cortex. a TLR7 transcript expression is present in all TSC specimens. Expression of TLRs 2, 4 and 7 was measured using the NanoString nCounter neuroinflammation assay and values for each specimen were normalized to the mean for the given TLR and shown in $\log 2$ scale. TLR7 was also quantified using qPCR. Expression levels were concordant between the two technologies $(P=0.0162$, Spearman's rho).

correlation is evident (Fig. 2b). IL-23A is a marker of TLR7 activation, so this pairwise relationship suggests that TLR7 activation in these tissues is linked to miR-142-3p levels.

To further examine TLR7 activation in TSC tubers, we queried our previous quantitative proteomics dataset derived from a comparison of onset/AMT-hot tubers and non-tuber control tissue [14]. Here, we calculated expression changes for nine proteins that are characteristic for TLR7 activation [13]. We found that eight were increased an average of 1.6-fold in epileptogenic tubers compared to control tissue $(P=0.0283$, Fig. 2 c). All TLRs (except TLR3) induce downstream signaling through the MyD88 adapter protein and stimulate two pathways: NF-kB and mitogen-activated protein kinases (MAPK). We found that median levels of MyD88 and MAPK1 are both increased in onset/AMT-hot tubers (Fig. 2d, e), and we previously reported NF-kB activation [14]. In $\mathrm{OH}$ tubers, 20 of 24 proteins known to be induced by NF-kB were significantly increased in expression $(P<0.0001$, Fig. 2f). Since TLRs 3 and 4 can cause subtle induction of IL-23A [9], we examined protein expression of two distinctive markers of TLR-3/4 activation: IFIT1 and MX1 [19]. These proteins were not significantly changed $(P>0.2)$, suggesting the observed IL-23A induction was driven by TLR7.

\section{Discussion}

Neuroinflammation is believed to be both an outcome and a contributor to recurrent seizures, although the molecular events in this cyclical relationship are poorly understood. We
Levels for the TLRs were not statistically significant when compared between categories. b Protein levels of signaling-competent TLR7 were detected in membrane fractions. Capillary electrophoresis Western analysis of membrane (M) and cytosolic (C) fractions was performed on two specimens for each tissue category. Calnexin was used as a marker specific to membrane fractions. Results are displayed as pseudo blots

previously linked increased miR-142-3p levels to inflammatory signaling in epileptogenic TSC tubers, as indicated by elevated AMT-PET uptake, yet the mechanism was unclear [6]. Another group concurrently demonstrated that miR$142-3 p$ is a ligand and potent activator of the TLR7 receptor [7]. That finding provided an important link to other studies showing TLR7 induces IDO1 and activates the kynurenine pathway, which is responsible for AMT uptake in epileptogenic lesions. Here, we established that TLR7 is indeed expressed in TSC tubers, demonstrating the capacity to signal through this pathway. However, the extent of TLR activation cannot be reliably inferred from receptor expression levels [20,21]. By examining characteristic downstream markers, we found evidence of TLR7 pathway activation in AMT-hot epileptogenic lesions. Importantly, IL-23A was significantly correlated to miR-142-3p levels, a known ligand and activator of TLR7.

The interaction among TLRs is complex and includes cooperative and antagonistic crosstalk. TLR7 has been shown to act synergistically with TLRs 2 and 4 [11]. Increased TLR4 expression was found in tissue of mesial temporal lobe epilepsy patients [22, 23]. Antagonistically, miR-142-3p has been shown to inhibit TLR4 activation [24], and TLR4 was reported to repress miR-142-3p expression [25]. The notably high levels of miR-142-3p in OH tubers suggest the dominant TLR pathway in this subset of lesions may be through TLR7. Further studies are needed to elucidate the relative roles of TLRs 2, 4 and 7 in the inflammatory response in epilepsy in TSC. Subsequent work may lead to opportunities for the use of neuroimaging and TLR7 inhibitors in a personalized approach to treating intractable 


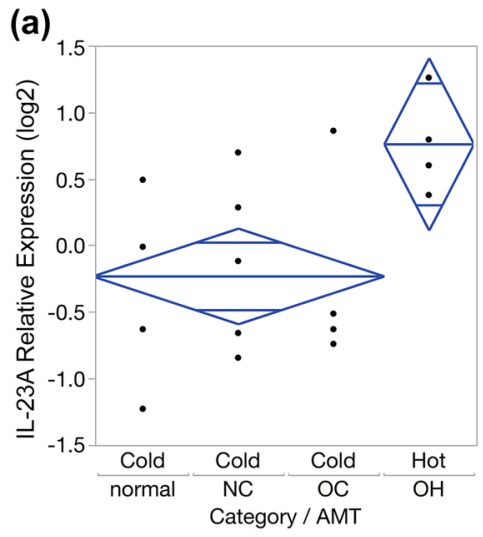

(d)

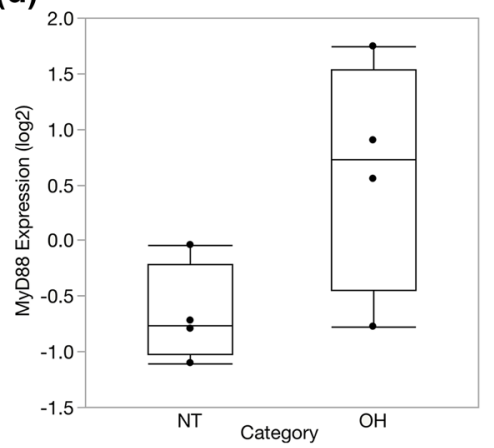

(b)

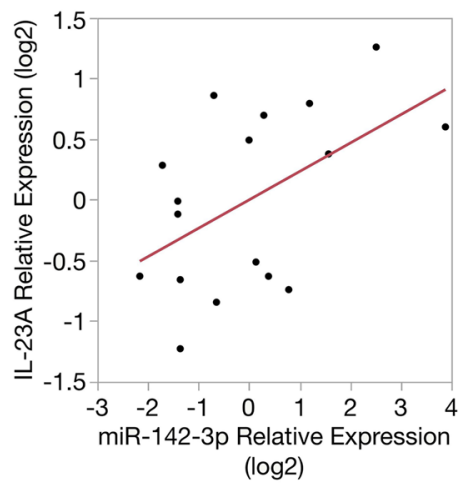

(e)

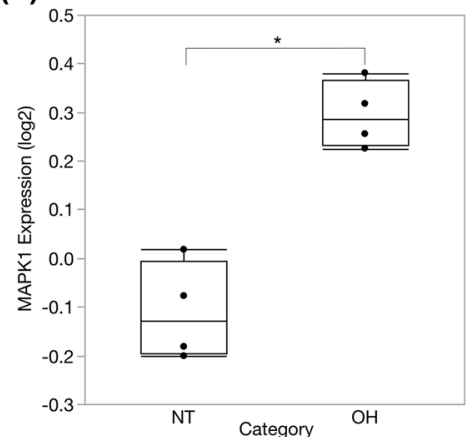

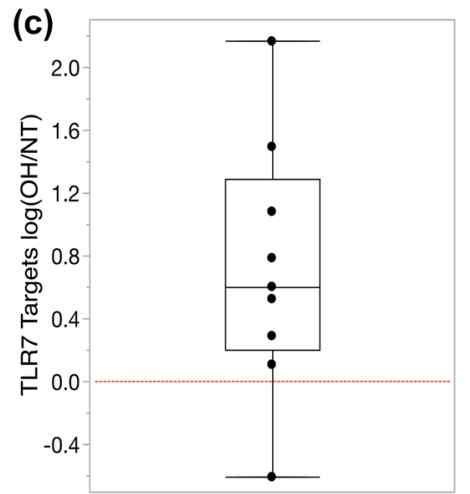

(f)

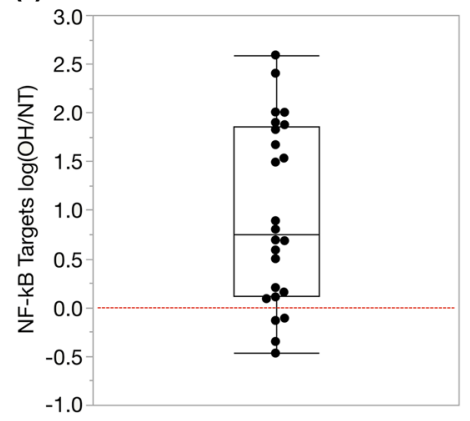

Fig. 2 Evidence of TLR7 pathway activation in AMT-hot epileptogenic TSC tubers. a IL-23A expression is an established marker of TLR7 activation. Using qPCR we measured expression of IL-23A in each category. Two-way ANOVA was used to investigate the relationship of IL-23A to AMT uptake and seizure onset status. IL$23 \mathrm{~A}$ is significantly associated with AMT uptake and increased in AMT-hot tubers $(\mathrm{OH}), P=0.0387, F$ ratio $=5.206$. 95\% confidence intervals (diamonds) are shown for AMT hot and cold groups, with mean values and overlap marks (horizontal bars near the tips of the diamonds). Vertical separation between the overlap marks of the two diamonds indicates statistical significance. $N=4,5,4,4$, respectively, for normal, $\mathrm{NC}, \mathrm{OC}$, and $\mathrm{OH}$ categories. b Regression analysis demonstrates that expression of miR-142-3p (a microRNA ligand and activator of TLR7) is a significant predictor of IL-23A mRNA levels in TSC tissue $(P=0.0318)$. c A set of proteins characteristic for TLR7 activation is significantly increased in $\mathrm{OH}$ tubers $(P=0.0283)$.

epilepsy. Importantly, drugs are currently available to inhibit the TLR7 pathway. The antimalarial drug chloroquine and derivatives inhibit activation of endosomal TLRs and are used for treatment of lupus [26]. Chloroquine crosses the blood brain barrier (BBB), and recent work has investigated the use of chloroquine for brain malignancies [27, 28]. A number of TLR7 antagonists are in clinical trials for treatment of autoimmune and inflammatory diseases such as rheumatoid arthritis, colitis, and multiple sclerosis [29-31].

Acknowledgements This work was supported by the National Institutes of Health grant R01 NS079429, Department of Defense CDMRP award TS130067, and Children's Hospital of Michigan Foundation
Proteins established as a signature of TLR7 activation [13] were analyzed using our previous quantitative proteomics dataset comparing $\mathrm{OH}$ vs normal control (NT) [14]. MS/MS spectra were available for nine signature proteins (C1QB, TRAFD1, HSPH1, TNS3, TAPBP, PSMB9, TAP2, TRADD, TNFAIP2). The difference in expression for each protein was calculated as $\log (\mathrm{OH} / \mathrm{NT})$. Positive values above the dashed line show increased expression for 8 of 9 proteins in $\mathrm{OH}$ specimens. $\mathbf{d}$ The median level of MyD88 protein is increased in $\mathrm{OH}$ tubers, albeit above statistical significance, $P=0.0665$. e MAPK1 protein, downstream of TLR7, is increased in $\mathrm{OH}$ tubers, $P=0.0006$. f A set of proteins known to be induced by NF-kB is significantly increased in $\mathrm{OH}$ tubers, $P<0.0001$. This observation was originally reported in [14]. Here, we present the change in expression for each $\mathrm{NF}-\mathrm{kB}$ target protein, calculated as $\log (\mathrm{OH} / \mathrm{NT})$. Positive values above the dashed line indicate increased expression for 20 of 24 $\mathrm{NF}-\mathrm{kB}$ target proteins in $\mathrm{OH}$ specimens

R2-2016-46. NanoString assays were contributed by NanoString Technologies and performed by the RTSF Genomics Core at Michigan State University. We would like to thank Dr. Aimee Luat and Dr. Eishi Asano at Wayne State University for their assistance with sample classification and Dr. Ron Thomas for statistical advice.

Open Access This article is distributed under the terms of the Creative Commons Attribution 4.0 International License (http://creativeco mmons.org/licenses/by/4.0/), which permits unrestricted use, distribution, and reproduction in any medium, provided you give appropriate credit to the original author(s) and the source, provide a link to the Creative Commons license, and indicate if changes were made. 


\section{References}

1. Vezzani A, Aronica E, Mazarati A, Pittman QJ. Epilepsy and brain inflammation. Exp Neurol. 2013;244:11-21.

2. Chugani HT, Kumar A, Kupsky W, Asano E, Sood S, Juhasz C. Clinical and histopathologic correlates of 11C-alpha-methylL-tryptophan (AMT) PET abnormalities in children with intractable epilepsy. Epilepsia. 2011;52:1692-8.

3. Chugani DC. Alpha-methyl-L-tryptophan: mechanisms for tracer localization of epileptogenic brain regions. Biomark Med. 2011;5:567-75.

4. Batista CE, Juhasz C, Muzik O, Kupsky WJ, Barger G, Chugani HT, et al. Imaging correlates of differential expression of indoleamine 2,3-dioxygenase in human brain tumors. Mol Imaging Biol. 2009;11:460-6.

5. Bosnyak E, Kamson DO, Guastella AR, Varadarajan K, Robinette NL, Kupsky WJ, et al. Molecular imaging correlates of tryptophan metabolism via the kynurenine pathway in human meningiomas. Neuro-Oncology. 2015;17:1284-92.

6. Bagla SCD, Asano E, Sood S, Luat A, Chugani H, Chugani D, Dombkowski A. A distinct microRNA expression profile is associated with alpha[11C]-methyl-L-tryptophan (AMT) PET uptake in epileptogenic cortical tubers resected from patients with tuberous sclerosis complex. Neurobiol Dis. 2018;109:76-87.

7. Feng Y, Zou L, Yan D, Chen H, Xu G, Jian W, et al. Extracellular microRNAs induce potent innate immune responses via TLR7/ MyD88-dependent mechanisms. J Immunol. 2017;199:2106-17.

8. Love AC, Schwartz I, Petzke MM. Induction of indoleamine 2,3-dioxygenase by Borrelia burgdorferi in human immune cells correlates with pathogenic potential. J Leukoc Biol. 2015;97:379-90.

9. Jensen SS, Gad M. Differential induction of inflammatory cytokines by dendritic cells treated with novel TLR-agonist and cytokine based cocktails: targeting dendritic cells in autoimmunity. J Inflamm (Lond). 2010;7:37.

10. Zurolo E, Iyer A, Maroso M, Carbonell C, Anink JJ, Ravizza $\mathrm{T}$, et al. Activation of toll-like receptor, RAGE and HMGB1 signalling in malformations of cortical development. Brain. 2011;134:1015-32.

11. Ghosh TK, Mickelson DJ, Solberg JC, Lipson KE, Inglefield JR, Alkan SS. TLR-TLR cross talk in human PBMC resulting in synergistic and antagonistic regulation of type- 1 and 2 interferons, IL-12 and TNF-alpha. Int Immunopharmacol. 2007;7:1111-21.

12. Bunger S, Roblick UJ, Habermann JK. Comparison of five commercial extraction kits for subsequent membrane protein profiling. Cytotechnology. 2009;61:153-9.

13. Guiducci C, Gong M, Cepika AM, Xu Z, Tripodo C, Bennett L, et al. RNA recognition by human TLR8 can lead to autoimmune inflammation. J Exp Med. 2013;210:2903-19.

14. Dombkowski AA, Batista CE, Cukovic D, Carruthers NJ, Ranganathan $\mathrm{R}$, Shukla U, et al. Cortical tubers: windows into dysregulation of epilepsy risk and synaptic signaling genes by microRNAs. Cereb Cortex. 2016;26:1059-71.

15. Smith PF. A guerilla guide to common problems in 'neurostatistics': essential statistical topics in neuroscience. J Undergrad Neurosci Educ. 2017;16:R1-12.

16. Petes C, Odoardi N, Gee K. The toll for trafficking: toll-like receptor 7 delivery to the endosome. Front Immunol. 2017;8:1075.
17. Hipp MM, Shepherd D, Gileadi U, Aichinger MC, Kessler BM, Edelmann MJ, et al. Processing of human toll-like receptor 7 by furin-like proprotein convertases is required for its accumulation and activity in endosomes. Immunity. 2013;39:711-21.

18. Maschalidi S, Hassler S, Blanc F, Sepulveda FE, Tohme M, Chignard $\mathrm{M}$, et al. Asparagine endopeptidase controls anti-influenza virus immune responses through TLR7 activation. PLoS Pathog. 2012;8:e1002841.

19. Doyle S, Vaidya S, O'Connell R, Dadgostar H, Dempsey P, Wu $\mathrm{T}$, et al. IRF3 mediates a TLR3/TLR4-specific antiviral gene program. Immunity. 2002;17:251-63.

20. Zarember KA, Godowski PJ. Tissue expression of human Tolllike receptors and differential regulation of Toll-like receptor mRNAs in leukocytes in response to microbes, their products, and cytokines. J Immunol. 2002;168:554-61.

21. Patni S, Bryant AH, Wynen LP, Seager AL, Morgan G, Thornton CA. Functional activity but not gene expression of toll-like receptors is decreased in the preterm versus term human placenta. Placenta. 2015;36:1031-8.

22. Pernhorst K, Herms S, Hoffmann P, Cichon S, Schulz H, Sander T, et al. TLR4, ATF-3 and IL8 inflammation mediator expression correlates with seizure frequency in human epileptic brain tissue. Seizure. 2013;22:675-8.

23. Leal B, Chaves J, Carvalho C, Rangel R, Santos A, Bettencourt A, et al. Brain expression of inflammatory mediators in mesial temporal lobe epilepsy patients. J Neuroimmunol. 2017;313:82-8.

24. Fordham JB, Naqvi AR, Nares S. Regulation of miR-24, miR$30 \mathrm{~b}$, and miR-142-3p during macrophage and dendritic cell differentiation potentiates innate immunity. J Leukoc Biol. 2015;98:195-207.

25. Sun Y, Sun J, Tomomi T, Nieves E, Mathewson N, Tamaki H, et al. PU.1-dependent transcriptional regulation of miR-142 contributes to its hematopoietic cell-specific expression and modulation of IL-6. J Immunol. 2013;190:4005-13.

26. Kuznik A, Bencina M, Svajger U, Jeras M, Rozman B, Jerala R. Mechanism of endosomal TLR inhibition by antimalarial drugs and imidazoquinolines. J Immunol. 2011;186:4794-804.

27. Firat E, Weyerbrock A, Gaedicke S, Grosu AL, Niedermann G. Chloroquine or chloroquine-PI3 K/Akt pathway inhibitor combinations strongly promote gamma-irradiation-induced cell death in primary stem-like glioma cells. PLoS One. 2012;7:e47357.

28. Osifo NG. The regional uptake of chloroquine in the rat brain. Toxicol Appl Pharmacol. 1979;50:109-14.

29. Wu YW, Tang W, Zuo JP. Toll-like receptors: potential targets for lupus treatment. Acta Pharmacol Sin. 2015;36:1395-407.

30. Hennessy EJ, Parker AE, O'Neill LA. Targeting toll-like receptors: emerging therapeutics? Nat Rev Drug Discov. 2010;9:293-307.

31. Lai CY, Su YW, Lin KI, Hsu LC, Chuang TH. Natural modulators of endosomal toll-like receptor-mediated psoriatic skin inflammation. J Immunol Res. 2017;2017:7807313.

Publisher's Note Springer Nature remains neutral with regard to jurisdictional claims in published maps and institutional affiliations. 